\title{
Influência do sombreamento na qualidade de mudas de Sclerolobium paniculatum Vogel para recuperação de área degradada
}

\section{Influence of shading on seedling quality of SclerolobiumpaniculatumVogel for recovery of degraded areas}

\author{
Gilson Araujo de Freitas ${ }^{1 *}$, Aurélio Vaz-de-Melo ${ }^{1}$, Mireia Aparecida Bezerra Pereira ${ }^{1}$, \\ Carlos Augusto Oliveira de Andrade ${ }^{1}$ e Guilherme Nunes Lucena', Rubens Ribeiro da \\ Silva ${ }^{1}$. \\ ${ }^{1}$ Departamento de Agronomia; Universidade Federal do Tocantins; 77402-970; Gurupi - TO - Brasil.
}

\begin{abstract}
The knowledge of the behavior of forest species in areas with different intensity of light, enables the definition and recommendation of the species in the recovery process. Thus, we aimed at verifying the influence of shading on seedling quality of Sclerolobiumpaniculatum Vogel in restoration projects of degraded areas. The experiment was conducted as a randomized block design with 15 replications. The treatments consisted of three levels of brightness (T1: 0\%, Full sun, T2: 50\%, Sombrite with 50\% retention of light and T3: Shadow Natural). Plants SclelorobiumpaniculatimVog.grown in an environment with 50\% sunlight had a growth rate in height ( $\beta 2: 0.0266)$ $40 \%$ higher than plants in full sun ( $\beta 2: 0.0161)$, most notably from the 103-day evaluation. Under natural shading had the lowest rate of development along the neck of the evaluation period, representing only 5.43\% ( $\beta 2: 0.0193)$ rate of development of plants under full sunlight ( $\beta 2: 0.3557)$. The increase in light intensity favors the major development of SclerolobiumpaniculatimVog. With that, may be indicated for use in projects aimed at recovery of degraded areas in the early successional pioneer and early secondary.
\end{abstract}

Key-words:shading,seedling quality,Sclerolobiumpaniculatum,revegetation

\section{INTRODUÇÃO}

Durante as últimas décadas, a Amazônia vem perdendo área de floresta para pastagens, exploração predatória de madeira e plantios de grãos. O reflorestamento de parte destas áreas, além dos benefícios ecológicos, aumentaria a oferta de madeira reflorestada na região e a renda na propriedade rural, diminuindo a pressão sobre as florestas naturais remanescentes (Toniniet al., 2005).

De acordo Oliveira Júnior (1997), a espécie florestal Sclerolobiumpaniculatum Vogel (taxibranco) possui rápido crescimento e elevada produção e desrama de folhas, o que favorece a formação de "litter". É uma espécie arbórea nativa de terra firme da região amazônica, pertencente à família leguminosae, subfmiliacaesalpinoideae. Assim, tais características conferem potencial para sua utilização em áreas sob processo de recuperação (Dias et al., 1995). Cabe destacar que a madeira produzida pelo taxi-branco apresenta características similares aos de eucalipto quanto ao poder calorífico da lenha e do carvão, bem como nos rendimentos de peso e volume no processo de carbonização ( Lorenzi, 2002).

A dinâmica de recuperação de uma floresta tropical é uma combinação de fatores, onde grupos de espécies com exigências complementares, principalmente quanto à necessidade de luz, são associados de tal forma que as espécies de estágios iniciais sejam sombreadoras das espécies de estágios finais em áreas sob recuperação. Esse

\footnotetext{
*Author for correspondence: araujoagro@hotmail.com
} 
fenômeno é conhecido como sucessão secundária e vêm servindo de base para distinguir os diferentes grupos ecológicos, bem como as espécies vegetais pertencentes a cada um dos grupos pioneiras, secundárias iniciais, secundárias tardias e clímax (Martins, 2009).

Dentre os grupos ecológicos, as espécies pioneiras e secundárias iniciais são as recomendadas para iniciarem os estágios sucessionais em projetos de recuperação de áreas degradadas, visto que essa recomendação é dada em função de sua adaptação as condições de maior luminosidade. As espécies pioneiras são dependentes de luz, não ocorrem em subosque, desenvolvem-se em clareiras ou em bordas de floresta e apresentam ciclo de vida menor que 10 anos. Já as espécies secundárias iniciais são mais tolerantes ao sombreamento, ocorrendo em clareiras pequenas, bordas de clareiras grandes, bordas de floresta ou subosque não densamente sombreado, com ciclo de vida de 10 a 25 anos (Ferretti et al., 1995; Carvalho, 2000).

Nos projetos de revegetação são plantadas entre 50 a $60 \%$ de espécies pioneiras, tolerantes a condições adversas e de crescimento rápido; 30 a $40 \%$ de secundárias iniciais e tardias, restando cerca de $10 \%$ de espécies clímax. Assim, a maior capacidade de adaptação das espécies às condições de alta insolação poderá garantir o sucesso de um projeto de recuperação de área degradada.

Características como altura, matéria seca, a relação raiz/parte aérea e diâmetro do colo da planta podem ser utilizadas para predizer o grau de tolerância das espécies em ambientes com diferentes intensidades luminosa (Carvalho et al., 2006). No entanto, deve se observar a qualidadedas mudas a serem implantadas.

Nesse sentido, o êxito na formação de florestas dealta produção depende, em grande parte, da qualidade das mudas plantadas, que, além de terem que resistir às condições adversas encontradas em campo após o plantio, deverão sobreviver e, por fim, produzir árvores com crescimento volumétrico economicamente desejável (Gomes et al., 1991). Qualidade são aqueles atributos necessários para que uma muda sobreviva e se desenvolva, após o plantio em campo.

Com isso, o objetivo foi verificar a influencia do sombreamento na qualidade de mudas de Sclerolobiumpaniculatum Vogelpara recuperação de área degradada.

\section{MATERIAL E MÉTODOS}

O trabalho foi conduzido na área experimental da Universidade Federal de Tocantins, Campus Universitário de Gurupi, localizado na região sul do Estado do Tocantins, a $280 \mathrm{~m}$ de altitude, nas coordenadas $11^{\circ} 43^{\prime} 45^{\prime \prime}$ de latitude e $49^{\circ} 04^{\prime} 07^{\prime \prime}$ de longitude. O clima regional é do tipo B1wA'a' úmido com moderada deficiência hídrica. A temperatura média anual é de $29,5{ }^{\circ} \mathrm{C}$, com precipitação anual de 1804 mm (Köppen, 1948).

$\mathrm{O}$ experimento foi conduzido em delineamento de blocos casualizados com 15 repetições, sendo a unidade experimental constituída por uma planta. Os tratamentos foram constituídos por três tratamentos de luminosidade (T1: 100\%, Pleno sol; T2: $50 \%$, Sombrite com $50 \%$ de retenção de luz e T3: Sombra Natural). No tratamento com sombra natural, as plantas foram colocadas sob uma área remanescente de vegetação nativa de cerrado Stricto sensu.

As sementes foram coletadas em plantas matrizes selecionadas quanto à sanidade vegetal após a dispersão de pelo menos $30 \%$ do volume total. Posteriormente a coleta, as sementes foram levadas ao Laboratório de Sementes da UFT/CAUG onde passaram por um protocolo de assepsia, e submetidas a um processo de expurgo.

A germinação das sementes foi realizada em canteiros contendo areia grossa lavada, localizado em viveiro com cobertura de sombritepermitindo a passagem de $50 \%$ da radiação solar. Após germinarem e atingirem o estádio de um par de folhas, as plântulas foram transplantadas para sacos de polietileno $(0,28 \mathrm{~m}$ de altura e $0,15 \mathrm{~m}$ de diâmetro), contendo aproximadamente $2 \mathrm{dm}^{-3} \mathrm{de}$ substrato na proporção $2: 1: 1$, sendo 2 : terra preta, coletado da camada superficial de cerrado nativo, 1: palha de arroz carbonizada e 1: substrato comercial PlantFlorest $\mathrm{I}^{\circledR}$ ), permanecendo por um período de 50 dias.

Após o período de formação, as plantas foram submetidas aos tratamentos por um período de aclimatação de 10 dias, sendo em seguida iniciadas as avaliações. A coleta dos dados foi realizada em intervalo de 21 dias, até os 145 dias após a emergência (DAE). As características avaliadas foram: taxa de crescimento em altura de planta (AP), taxa de crescimento em diâmetro de colo (DC), massa seca de folhas (MSF), massa seca de caule (MSC), massa seca de raízes (MSR) e massa seca total (MST), Razão da massa foliar (RMF), Razão da massa caulinar (RMC), Razão da massa radicular (RMR), razão parte aérea/raiz 
(PA/R), Partição da massa seca (DEMS) e índice de qualidade do desenvolvimento das mudas (IQD). A altura foi considerada desde o colo até o ápice de cada planta, medida com régua graduada em centímetros $(\mathrm{cm})$, e o diâmetro do colo medido com paquímetro graduado em milímetros $(\mathrm{mm})$, tomado a um centímetro do solo.

$\mathrm{Na}$ obtenção do Índice de Qualidade do Desenvolvimento (IQD) foi utilizada a metodologia de Dicksonet al. (1960), considerando os indicadores de massa seca da parte aérea, das raízes e de massa seca total, altura e diâmetro do colo das mudas, conforme a equação 1 :

Onde:

$$
I Q D=\frac{M S T(g)}{\frac{H(c m)}{D C(c m)}+\frac{P M S P A(g)}{P M S R A(g)}}
$$

IQD = Índice de desenvolvimento de Dickson, MST = Massa seca total $(\mathrm{g})$,

$\mathrm{H}=$ altura $(\mathrm{cm}), \mathrm{DC}=$ diâmetro do colo $(\mathrm{cm})$, PMSPA = Peso da matéria seca da parte aérea (g) e PMSRA = Peso da matéria seca da raiz $(\mathrm{g})$. A fórmula pode ser entendida, como: Quanto maior o índice de IQD, melhor os resultados. A Planta deve ser baixa, com colo grosso, menos folhas e mais raiz.

Para a determinação da massa seca as plantas foram separadas em folhas, caule e raízes. $\mathrm{Na}$ remoção do substrato aderido as raízes foram utilizados jatos brandos de água. Esta operação foi realizada sobre peneira com abertura de $2 \mathrm{~mm}$, de forma a evitar a perda de raízes (Moraes Neto et al., 2000). A obtenção das massas foi realizada separadamente em balança eletrônica analítica e acondicionado em sacos de papel devidamente identificados, os quais foram colocados em estufa com circulação de ar forçada, à aproximadamente $70{ }^{\circ} \mathrm{C}$, durante 72 horas.

Com os resultados obtidos nas avaliações da altura de planta e diâmetro de colo foram realizadas as análises de regressões em função do tempo, as demais caracteristicas foram submetidas a análise de variância, e as médias comparadas pelo teste tukey $5 \%$.

\section{RESULTADOS E DISCUSSÃO}

Plantas de Sclelorobiumpaniculatim vog. cultivadas em ambiente com 50\% de luminosidade apresentaram uma taxa de crescimento em altura $\left(\beta_{2}: 0,0266\right) 40 \%$ maior do que as plantas em pleno sol $\left(\beta_{2}: 0,0161\right)$, com maior destaque a partir dos 103 dias de avaliação (Figura 1). Na condição de sombra natural as plantas apresentaram maior altura inicial até os 93 dias. Após esse período as plantas apresentaram altura inferior àquelas submetidas às condições de maiores luminosidades. Siebeneichleret al. (2008) avaliando a espécie Tabebuia heptaphyilla(Vell.) e Silva et al. (2007) avaliando a espécie Theobromagrandiflorum (Willd. ExSpreng.) Schum também verificaram maior desenvolvimento inicial quando as plantas foram expostas a condição de $50 \%$ de luminosidade e pleno sol. Resultados semelhantes foram observados com castanha do maranhão (Bombacopsis glabra), (Scalonet al., 2003), canela-batalha (Criptocariaaschersoniana), (Almeida et al., 2004) e Macluratinctoriaee Senna macranthera(Almeida et al., 2005). Todavia, isso não ocorreu em Hymenaeaourbarilee Acaciamangium(Almeida et al., 2005) e Cedrelafissilis(Santos et al., 2006), quando comparado com plantas mantidas a pleno sol. Isso mostra a existência de uma diversidade das espécies florestais quanto ao seu comportamento em diferentes ambientes. Dessa forma a luminosidade é considerada como um fator seletivo na definição da comunidade vegetal durante os estágios sucessionais em áreas sob recuperação.
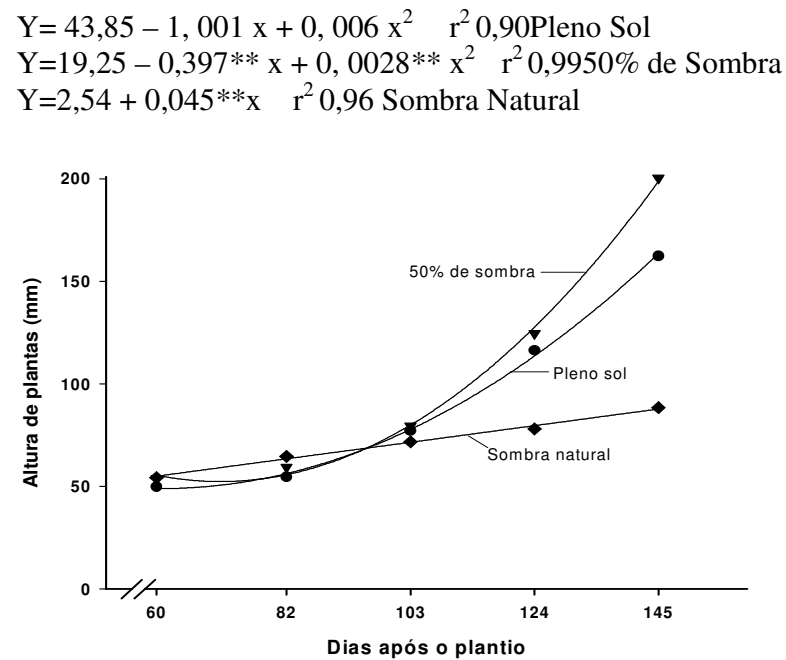

Figura 1-Altura de plantas de Sclelorobiumpaniculatim Vog. em função em função do tempo.

As plantas Sclelorobiumpaniculatim vog. expostas em ambiente com sombra natural apresentaram os menores incrementos em alturas ao longo do tempo (Figura 1), provavelmente em função da menor disponibilidade de fotoassimilados para o 
seu crescimento, já que sob sombra natural a taxa fotossintética deveria ser muito baixa em relação às plantas cultivadas nos ambientes com maior disponibilidade de luz (Taize Zieger, 2002).

$\mathrm{Y}=-1,65+0,354 * * \mathrm{xr}^{2} 0,85$ Pleno Sol

$\mathrm{Y}=2,25-0,045 * \mathrm{x}+0,0004 * * \mathrm{x}^{2} \quad \mathrm{r}^{2} 0,9950 \%$ de Sombra $\mathrm{Y}=0,75+0,0025 * * \mathrm{x} \quad \mathrm{r}^{2} 0,95$ Sombra Natural

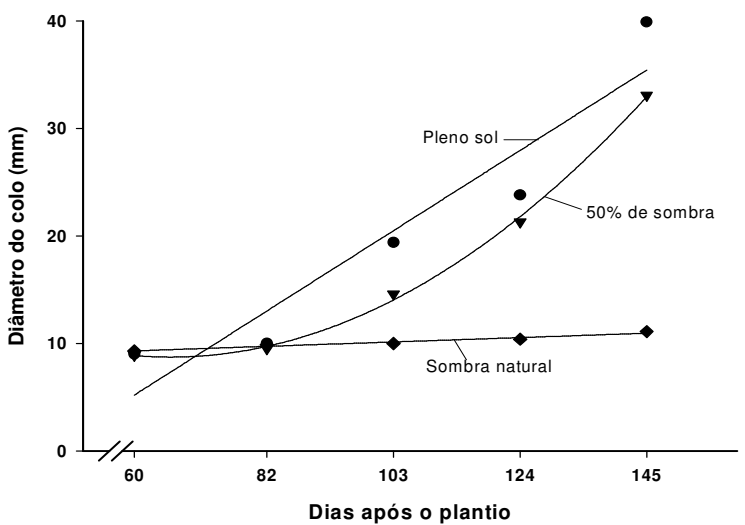

Figura 2- Diâmetro do colo de plantas de

Sclelorobiumpaniculatim Vog. em função do tempo.

As plantas sob sombreamento natural apresentaram as menores taxa de desenvolvimento do colo ao longo do período de avaliação, representando apenas 5,43\% $\left(\beta_{2}: 0,0193\right)$ da taxa de desenvolvimento das plantas submetidas a pleno sol $\left(\beta_{2}\right.$ : 0,3557). Contudo, a taxa de desenvolvimento do colo da planta quando submetida ao ambiente com 50\% de luminosidade apresentou comportamento quadrático, sendo observado um aumento significativo na taxa de desenvolvimento a partir dos 82 dias(Figura 2). Entretanto, é possível perceber uma tendência de maior desenvolvimento das plantas submetidas a 50\% de luminosidade após os 145 dias em relação àquelas em pleno sol.

Almeida et al. (2005), trabalhando com Acaciamangium e Siebeneichleret al. (2008) avaliando a espécie Tabebuia heptaphyilla(Vell.) verificaram redução no diâmetro do colo em plantas com o aumento do sombreamento. Todavia, Almeida et al. (2005) trabalhando com Macluratinctoriaee Hymenaeacourbaril e Silva et al. (2007) avaliando a espécie Theobromagrandiflorum (Willd. ExSpreng.) Schum não verificaram diferenças no desenvolvimento do colo da planta sob $50 \%$ de sombreamento em relação a mudas cultivadas a pelo sol. Em algumas espécies maior luminosidade permite uma taxa fotossintética mais elevada, logo maior acúmulo de fotoassimilados no caule das plantas (Taiz e Zieger, 2002).Mais fotoassimilados, comparando ao ambiente de sombra natural, pode ser observado na espécie Sclelorobiumpaniculatim, pois o crescimento dasplantaspode refletir a habilidade de adaptação das espécies às condições de radiação do ambiente em que estão se desenvolvendo.A seleção de espécie florestal com essa característica como a Sclelorobiumpaniculatim vog. viabiliza a sua utilização como espécie pioneira em projetos de recuperação da vegetação em áreas degradadas.

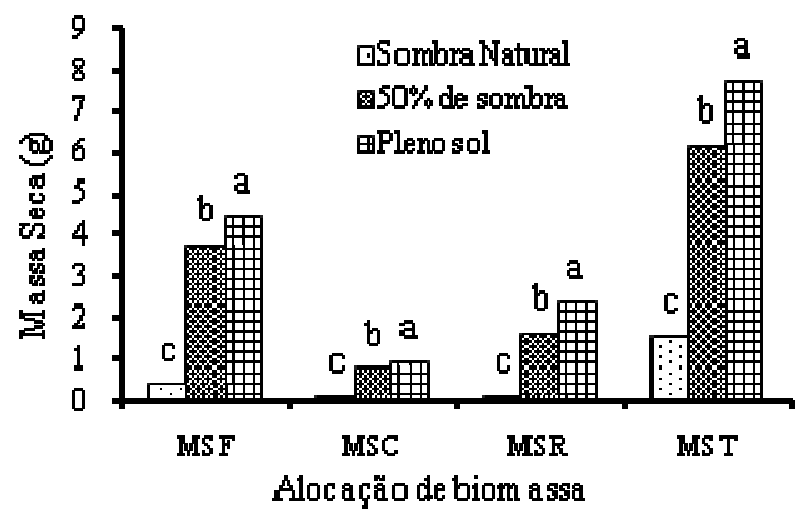

Figura 3 - Massa Seca da Folha (MSF), Massa Seca do Caule (MSC), Massa Seca de Raiz (MSR) e Massa Seca Total (MST) das plantas de Sclelorobium paniculatim $\mathrm{Vog}$, em função dos níveis de sombreamento, aos 145 dias de idade.

Plantas submetidas a ambientes de pleno sol apresentaram maior peso de massa seca de folha, caule, raiz e total (Figura 3). Todas as características avaliadas apresentaram incrementos de massa com o aumento da intensidade luminosa. Provavelmente as plantas aumentaram a fotossíntese sob maior disponibilidade de luz, ocorrendo um incremento no teor de carboidratos nas folhas, caule e raiz que influenciou na elevação da massa seca (Larcher, 2000). Resultados semelhantes foram encontrados em duas espécies de carvalho sob duas condições de luminosidade, apresentando melhor desempenho das plantas a pleno sol em termos de matéria seca total, de raízes, caules e folhas (KeendWerger, 1999). Costa et al. (2011) observaram em plantas de Jatrophacurcas, que houve maior acúmulo de massa seca em folhas e massa seca totalem plantas submetidas ao tratamento com $50 \%$ de sombreamento.

Os níveis de sombreamento não ocasionaram diferença significativa para as razõesRMF, RMC e RMR(Figura 4). No entanto, houve tendência de 
maior razão de massa foliar. Isto significa que, à medida que a planta cresce, maior é a fração do material retido na folha, ou seja, a exportação paraoutras parte é menor.A exportação é uma característica genética influenciada pelo ambiente. Quanto maior o valor da razao da massa da folha , maior é o investimento da planta em produção de folhas. Componente basicamente fisiológico, a Razão da Massa Caulinar (RMC) expressa a fração da massa seca que não foi exportada do caule para o resto da planta. Enquanto a RMR expressa a parcela de massa seca não exportada das raizes para o resto da planta.Considerando-se que as folhas são os centros de produção de massa da matéria seca, mediante a fotossíntese, e que o resto da planta depende da exportação de material da folha, a RMF expressa a fração de massa da matéria seca não exportada das folhas para o resto da planta (Benincasa, 1988).

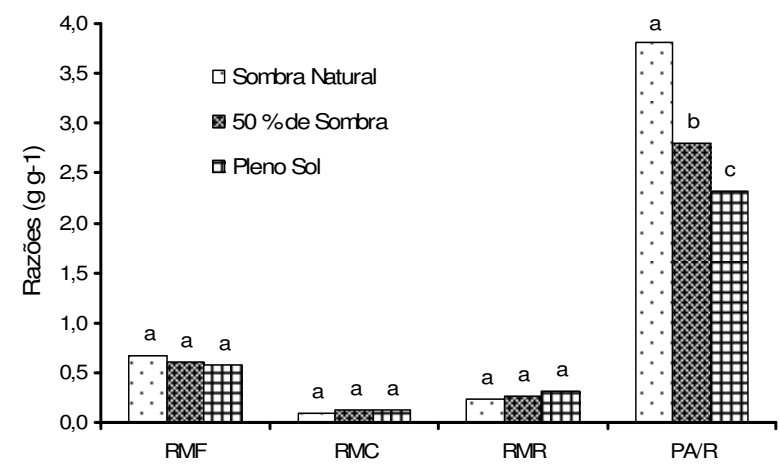

Figura 4- Razão da Massa Foliar (RMF), Razão da Massa Caulinar (RMC), Razão da Massa Radicular (RMR) e Razão Parte Aérea/Raiz (PA/R) de plantas de Sclelorobiumpaniculatim Vog, em função dos níveis de sombreamento.

Contudo, houve diferença significativa para a relação parte aérea/raiz (Figura 4). A menor relação parte aérea/raiz em plantas sob alta intensidade de luz indica maior alocação de fotoassimilados no sistema radicular. Plântulas com sistema radicular bem desenvolvido têm maiores chances de sobrevivência no campo. O desequilíbrio pode ser prejudicial em termos de adaptação após o plantio em local definitivo. Além disso, plântulas com crescimento em altura excessivo podem sofrer tombamento, resultando em alteração do padrão de qualidade planta adulta. De acordo Carvalho et al. (2006), essa é uma estratégia que permite a planta realizar maior absorção de água e nutrientes para suportar altas taxas de fotossíntese e transpiração sob alta intensidade de luz. Plantas com esse tipo de estratégia devem ser sugeridas para o uso em áreas sob processo de recuperação, uma vez que podem garantir o desenvolvimento inicial e condicionar a continuidade dos estágios sucessionais, sendo este o caso da espécie SclelorobiumpaniculatimVog.

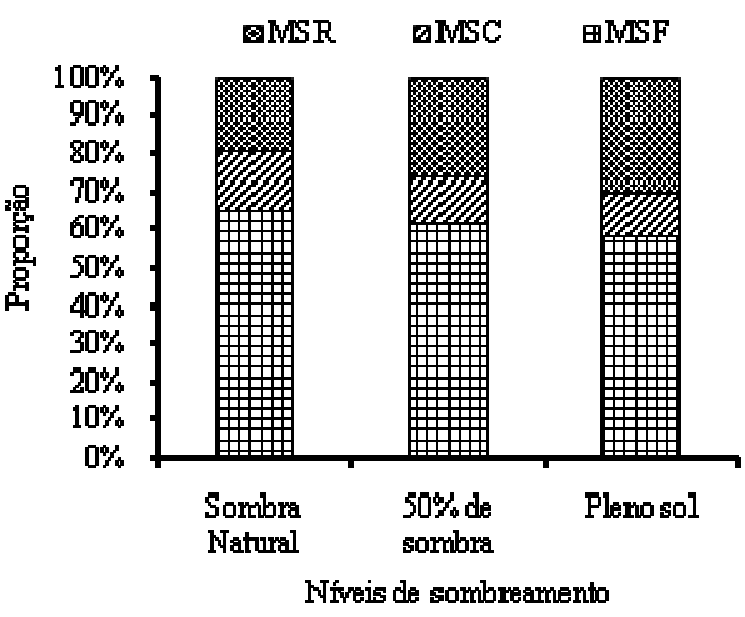

Figura 5- Distribuição espacial da massa seca de plantas de Sclelorobium paniculatim em folhas (MSF), caule (MSC) e raízes (MSR) em função de níveis de sombreamento.

Na avaliação da partição da massa seca de plantas de Sclelorobiumpaniculatimvog, foi verificado o aumento da produção de raiz com a intensidade de luz, assim como redução na produção de caule e folhas (Figura 5). Provavelmente, o aumento da intensidade luminosa, tenha favorecido o aparato fotossintético da planta, na qual direcionou maior quantidade de fotoassimilados para o sistema radicular. Silva et al. (2007), estudando o desenvolvimento inicial da espécie Hymenaeaparvifolia também verificou menor produção de raiz em função da redução na intensidade de luz. Ao contrário das espécies citadas a Theobromagrandiflorumapresentou maior produção de raiz sob a condição de $50 \%$ de intensidade luz (Silva et al., 2007). Lee et al. (1996), cultivando plantas em baixa intensidade de luz observou que essas tendem a investir mais na produção de biomassa da parte aérea em detrimento da raiz.

$\mathrm{O}$ índice de qualidade do desenvolvimento das mudas (IQD) de Sclelorobiumpaniculatim vog. sob os diferentes níveis de sombreamento obteve resposta significativa. Foi observado que à medida que houve diminuição do nível de sombreamento, 
ou seja, aumento da incidência luminosa, as plantas apresentaram qualidade do desenvolvimento superior (figura 6).

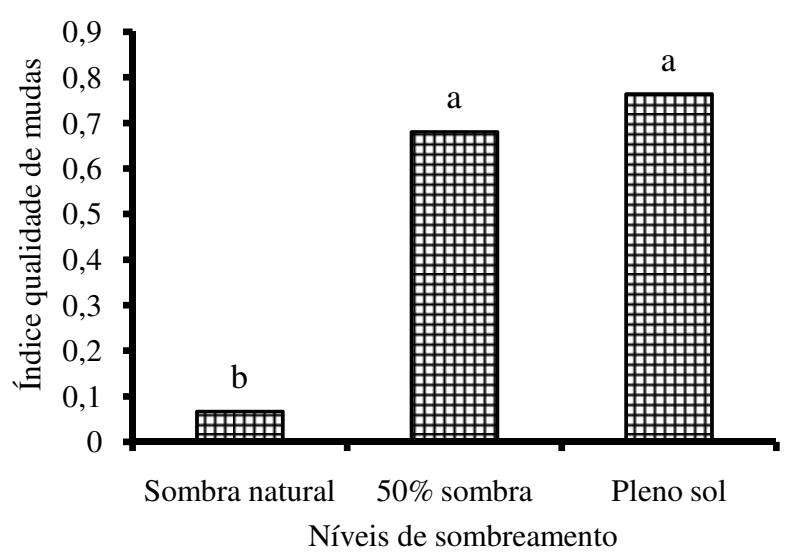

Figura 6-Índice de qualidade do desenvolvimento de mudas de Sclelorobiumpaniculatim em função dos níveis de sombreamento em, aos 145 dias de idade.

O IQD é apontado como bom indicador da qualidade de mudas, por considerar para o seu cálculo a robustez e o equilíbrio da distribuição da fitomassa das mudas, sendo ponderados vários parâmetros importantes (Fonseca, 2000). Estudando o padrão de qualidade de mudas de Trema micrantha (L.) Blume, produzidas sob diferentes períodos de sombreamento, Fonseca et al. (2002) avaliaram os parâmetros morfológicos das mudas, suas relações e o índice de qualidade de Dickson. As mudas desenvolvidas sob maiores períodos de sombreamento, embora tenham alcançado maiores alturas das partes aéreas e áreas foliares, apresentaram as piores qualidades, com redução do diâmetro do coleto, da fitomassa seca do sistema radicular e do índice de qualidade de Dickson e aumento da relação altura da parte aérea/diâmetro do coleto e da relação parte aérea/sistema radicular. seca do sistema radicular e do índice de qualidade de Dickson e aumento da relação altura da parte aérea/diâmetro do coleto e da relação parte aérea/sistema radicular.

Outros estudos foram realizados com espécies florestais, estabelecendo como padrão de IQD o valor mínimo de 0,20 (Hunt, 1990). Observa-se neste trabalho que as plantas submetidas a $50 \%$ de sombra e pleno sol apresentaram valores de IQDde 0,68 e 0,76 respectivamente, mostrando que as mudas deSclelorobiumpaniculatim produzidas nesses dois níveis de sombreamento apresentam qualidade para plantio.Em relação às mudas acondicionadas no ambiente de sombra natural, o IQD foi inferior a 0,20 não devendo ser indicado para plantio, devido ter baixa qualidade de desenvolvimento.

\section{CONCLUSÕES}

$\mathrm{O}$ aumento da intensidade de luz favorece o maior desenvolvimento das plantas de Sclerolobiumpaniculatum Vog.

As plantas de Sclerolobiumpaniculatum Vog. podem ser indicadas para o uso em projetos que visam de recuperação de áreas degradadas, tanto para as condições de pleno sol, quanto para $50 \%$ de sombreamento das mudas.

\section{REFERÊNCIAS}

Almeida, I. P .; Alvarenga, A. A.; Castro, E. M.; Zanela, S. M.; Vieira, C. V. (2004), Crescimento inicial de CryptocariaaschersonianaMez. Submetida a níveis de radiação solar. Ciência Rural, Santa Maria, 34, 83-88.

Almeida, S. M. Z. ; Soares, A. M.; Castro, E. M.; Vieira, C. V.; Gajego, E. B. (2005), Alterações morfológicas e alocação de biomassa em plantas jovens de espécies florestais sob diferentes condições de sombreamento. Ciência Rural, 35,62-68.

Benincasa, M. M. P. Análise de crescimento de plantas: noções básicas. Jaboticabal: FUNEP, 2003, 42 p.

Carvalho, N. O. S.; Pelacani, C. R.; Rodrigues, M. O. S.; Crepaldi, I. C. (2006), Initialgrowthoflicuriplants

(Syagruscoronata(Mart.) Becc.) under different light intensity. Revista Árvore, 30, 351-357.

Carvalho, P. E. R. Produção de mudas de espécies nativas por sementes e a implantação de povoamentos. In: Galvão, A. P. M. (Org.). Reflorestamento de propriedades rurais para fins produtivos e ambientais: um guia para ações municipais e regionais. Brasília: Embrapa, 2000. p.151-174.

Costa, J. L.; Lima, R. P.; Silva, A. L. L.; Scheidt, G. N.; Erasmo, E. A. L. (2011),Crescimento inicial de plantas de pinhão manso em função do sombreamento no município de Gurupi-TO. 
JournalofBiotechnologyandBiodiversity,2,43-47, 2011

Dias, L. E.;Brienza Junior, S.; Pereira C.A. (1995), Táxi branco (SclerolobiumpaniculatumVogel): Uma leguminosa arbórea nativa da Amazônia com potencial para recuperação de áreas degradadas. In: Kanasiro, M.; Parrota, J.A. Manejo e reabilitação de áreas degradadas e florestais secundarias na Amazônia. Paris, France: UNESCO, p. 148-153.

Ferretti, A.R.; Kageyama, P.Y.;Arboez, G.F.; Santos, J.D.; Barros, M.I.A.; Lorza, R.F.; Oliveira,C. (1995),Classificação de espécies arbóreas em grupos ecológicos para revegetação com nativas no Estado de São Paulo. Florestar Estatístico, 3,73-77.

Fonseca, E. P.; Valéri, S. V.; Miglioranza, E.; Fonseca, N. A. N.; Couto, L. (2002),Padrão de qualidade de mudas de Trema mícrantha (L.) Blume, produzidas sob diferentes períodos de sombreamento. Revista Árvore, 26,515-523.

Fonseca, E.P. Padrão de qualidade de mudas de Trema mícrantha (L.) Blume, Cedrelafissilis Veli. eAspidospermapolyneuronMüll Arg. produzidas sob diferentes períodos de sombreamento. Tese (Doutorado em Agronomia) - Universidade Estadual Paulista, Jabotical, 2000.

Gomes, J. M.; Couto, L.; Gonçalves, R. D. E. C. (1991), Efeito de diferentes substratos na produção de mudas de Eucalyptusgrandis W. Hill exMaiden, em "Win-Strip". Revista Árvore, 15,35-42.

Hunt, G.A. Effect of styroblock design and cooper treatment on morphology of conifer seedlings. In: TARGET SEEDLINGS SYMPOSIUM, MEETING OF THE WESTERN FOREST NURSERY ASSOCIATIONS, Roseburg, 1990. Proceedings... p. 218-222. Fort Collins: United States Departament of Agriculture, Forest Service, 1990. (RM-GTR-200).

Ke, G. andWerger, M.J.A.(1999), Different responses to shade of evergreen and deciduous oak seedlings and the effect on acorn size. Acta Oecologica, 20,579-586.

Larcher, W. Ecofisiologia Vegetal: São Carlos, rima. 531p. 2000 .
Lee, D. W.; Baskaran, K.; Mansor, M.; Mohamad, H.; Y. A. P., S.K. (1996), Irradiance and spectral quality affect Asian tropical rain forest tree seedling development. Ecology, 77, 568-580.

Lorenzi, H. Árvores brasileiras: manual de identificação e cultivo de plantas arbóreas nativas do Brasil. 4. Ed. Nova Odessa: Plantarum, v. 1, 368p, 2000.

Martins, S.V. Recuperação de áreas degradadas: ações em áreas de preservação permanente, voçorocas, taludes rodoviário e de mineração. Viçosa, MG. 270p, 2009.

Moraes Neto, S.P.; Gonçalves, J.L. De M.; Takaki, M.; Cenci, S.; Gonçalves, J.C. (2000), Crescimento de mudas de algumas espécies arbóreas que ocorrem na mata atlântica, em função do nível de luminosidade. Revista Árvore, 24, 3545 .

Oliveira Júnior, V.M.Crescimento e nutrição mineral de Táxi-branco-de-terra-firme (SclerolobiumpaniculatumVogel) micorrizadas e adubadas com diferentes fontes de fosfato natural. Dissertação(Mestrado em Ciências Florestais) Faculdade de Ciências Agrárias do Pará, 1997.

Santos, D. L.; Rakocevic, M.; Takaki, M.; Ribaski, J. (2006), Morphological and physiological responses of CedrelafissilisVellozo (Meliaceae) seedlings to light. BrazilianArchivesofBiologyand Technology,49,171-182.

Scalon, S.P.Q.; Mussury, R.M.; Rigoni, M.R.; Filho, H.S. (2003), Crescimento inicial de mudas de Bombacopsis glabra (Pasq.) A. Robinssob condição de sombreamento. Revista Árvore, 27, 753-758.

Siebeneichler, S. C.; Freitas, G.A. Silva, R.R.; Adorian, G.C.; Capellari, D. (2008),Características morfofisiológicas em plantas de Tabebuia heptaphyilla (vell.) tol. em condições de luminosidade.Acta Amazonica. 38,467-472.

Silva, B. M.S.; Lima, J. D. L.; Dantas, V.A.V.; Moraes, W. S.; Sabonaro, D. Z. (2007),Efeito da luz no crescimento de mudas de HymenaeaparvifoliaHuber.Revista

Árvore,31,1019-1026. 
Silva, R. R.; Freitas, G. A.; Siebeneichler, S. C.; Mata, J. F.; Chagas, J. R. (2007), Desenvolvimento inicial de plântulas de Theobromagrandiflorum (Willd. exSpreng.) Schum. sob influência de sombreamento.Acta Amazonica, 37,365-370.

TaizandZieger.Plant physiology, 3.Ed. Sunderland: Sinauer Associates inc. 690p. 2002.
Tonini, H.; Pereira, M.R.N.; Arco-Verde, M.F.; Oliveira Junior, M.M. (2005),Seleção de equações para o paricá (Schizolobiumamazonicum Huber exDucke), no estado de Roraima. Boletim de Pesquisa e Desenvolvimento,04, 1-17. 